\title{
Residual Gas Components and Their Effect on Field Emission Devices with Nanoscale Emitters
}

\author{
Jinchan WANG*1,†1, Wei TIAN*1, Ligong SUN*1, Bo LIU*1, Leiming ZHANG*1 and Lu SONG*1 \\ ${ }^{*}$ College of Electrical Engineering, Henan University of Science and Technology, Luoyang, P. R. China 471023
}

(Received March 14, 2014, Accepted September 4, 2014)

\begin{abstract}
The emission currents of zinc oxide ( $\mathrm{ZnO}$ ) and carbon nanotube (CNT) cold cathodes show continuous degradation in sealed field emission (FE) devices when the anode voltages are maintained at a constant value; more stable currents are observed when the same cathodes are operated under ultra-high vacuum (UHV) conditions. This shows that ambient gases in the devices play an important role in degrading the emission currents of $\mathrm{ZnO}$ and CNT cathodes. To investigate the gas conditions in sealed FE devices, a vacuum system with a quadrupole mass spectrometer (QMS) was employed here. QMS data obtained before and af-

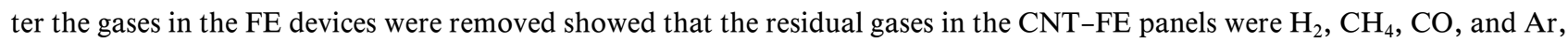
while in the $\mathrm{ZnO}-\mathrm{FE}$ panels the residual gases were $\mathrm{H}_{2}, \mathrm{CH}_{4}, \mathrm{CO}, \mathrm{Ar}$, and $\mathrm{CO}_{2}$. It was clear that the residual gases in the two kinds of FE devices were slightly different; this was mainly due to the different cathode materials used in the FE devices. The residual gases affected the emission current of the $\mathrm{CNT}$ and $\mathrm{ZnO}$ FE devices by changing the work function at the cathode surface; this was confirmed by the F-N curves measured when the same cathodes were tested in the devices and in the UHV chamber.
\end{abstract}

\section{Introduction}

Nanostructured materials are considered efficient field emitters for field emission devices (FEDs) and related vacuum microelectronic devices. The reduced dimensions of the nanomaterials result in high local electric fields, and large emission currents can be achieved ${ }^{1)}$. In the last few years, carbon nanotubes and zinc oxide have been applied as cathode materials for field emission applications ${ }^{2,3)}$. The simplicity of the methods required for the synthesis of $\mathrm{CNT}$ and $\mathrm{ZnO}$ mean that these materials have been widely applied in field emission devices. Much attention has been paid to the FE properties of CNT and $\mathrm{ZnO}$, motivated especially by the fact that screen printing methods have been proposed for the low-cost fabrication of large-area FE cathodes.

The field emission from CNTs and $\mathrm{ZnO}$ can be described by the Fowler-Nordheim $(\mathrm{F}-\mathrm{N})$ equation ${ }^{4}$

$$
\ln \left(\frac{I}{V^{2}}\right)=\ln \left(A a \frac{\beta^{2}}{\Phi}\right)-B\left(\frac{\Phi^{3 / 2}}{\beta}\right)\left(\frac{1}{V}\right),
$$

where $\mathrm{I}$ is the emission current, $A$ and $B$ are constants, $\alpha$ is the emission area, $\Phi$ is the work function, $V$ is the applied voltage, and $\beta$ is the geometric field enhancement factor. This equation shows that the emission current of the emitters depends strongly on the work function and geometry of the cathode surface.

For the application of CNTs and $\mathrm{ZnO}$ in commercial FEDs to be feasible, long-term current stability must be ensured. Although CNTs and ZnO have been reported as the most likely candidate for $\mathrm{FE}$ devices, some investigations ${ }^{5-9)}$ have reported emission current degradation or failure in these materials. One of the causes for this deterioration is an excessive residual gas pressure inside the sealed FEDs; FEDs require a high vacuum for

†1 E-mail: wang4712279@163.com, Phone: +86-379-64231910 their operation ${ }^{10-12)}$. The presence of these residual gases in these devices can be due to a number of factors, including the outgassing of emitters and other device materials, residues from the fabrication process, electron-stimulated desorption from surfaces that are bombarded by electrons or positive ions, phenomena associated with extremely high electric fields, dissociation and ion sputtering, and the electron-impact ionization of gases between cathode and anode ${ }^{13)}$. These residual gases can influence the field emission current of the FE cathode $^{14)}$ via adsorption, chemical reactions, or ion bombardment, therefore affecting the emission stability of the cathode. It is therefore important to analyze whether the residual gases do in fact affect the emission current stability of the cathodes and the residual gas components inside sealed FEDs. However, few reports have focused on the real working conditions for CNT and $\mathrm{ZnO}$ cathodes.

In this work, an experimental system was set up to measure the emission current stability of the same cathode in a sealed FE device and in a high vacuum (UHV) system, and the ambient gas conditions in sealed CNT-FE and ZnO-FE devices were analyzed.

\section{Experimental}

The FE-devices used in the experiments were of the diode type, and they were provided with evaporated Ba-getters. A metal-on-glass layer was employed as the anode substrate. The screen-printing method was adopted to deposit the CNT or $\mathrm{ZnO}$ paste on the cathode substrate. The $\mathrm{ZnO}$ or CNT paste was a mixture of organic binder material, organic solvent, and multi-wall CNTs or tetrapod $\mathrm{ZnO}$. A scanning electron microscope (SEM) was used to characterize the surface morphology of the printed film. Figure 1 shows the surface morphologies of an as-prepared CNT-cathode and a $\mathrm{ZnO}$-cathode. The anode and cathode glass plates were then sealed using a 


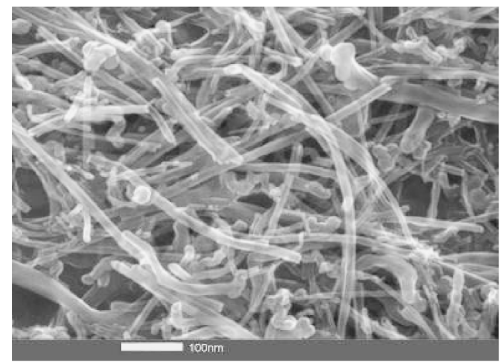

(a)

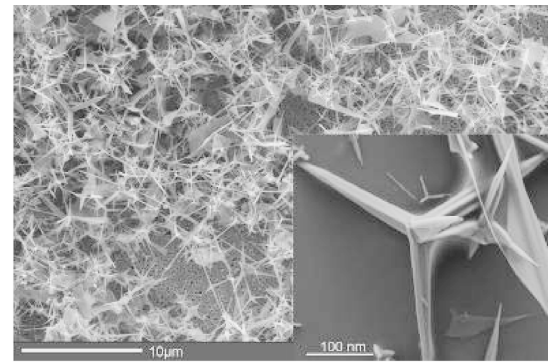

(b)

Fig. 1 Surface morphology of an as-prepared CNT-cathode (a), and a ZnO-cathode (b)

conventional glass frit, at a temperature of $430^{\circ} \mathrm{C}$, in air. After this, the diode device was pumped at a pressure of $3 \times 10^{-4} \mathrm{~Pa}$ for approximately 2 hours at a temperature of $340^{\circ} \mathrm{C}$, and was sealed after the pumping process. The device was then installed on the vacuum system using an opening set (consisting of a metal tube with a glass breaking tool) that was mounted on the anode plate.

\section{Results and Discussion}

To study whether the ambient gases affected the emission current stability of the CNT and ZnO cathodes, it was necessary to use the same cathode in different experiments. In addition, the other working conditions were kept constant while the gas conditions inside the device were varied, so that other factors that might also have led to the emission current degradation of the cathode could be avoided. Thus, only the influence of the residual gases was observed. Following these considerations, an experimental system was set up as shown in Fig. 2. It consisted of a vacuum system with a quadrupole mass spectrometer (QMS), the FE device, and the testing apparatus. The vacuum system was composed of a 1.51 stainless steel vacuum chamber, a sputter ion pump, a UHV ionization gauge (B-A gauge), two UHV valves, and connection tubes. The experiments could be carried out at 400 ?C, but typically, lower temperatures were used. A mechanical pump and a turbo molecular pump were used for the initial pumping down to the high-vacuum range in the chamber. The total pressure in the chamber was measured using a conventional UHV ionization gauge (B-A gauge). The pressures measured with the B-A gauge are given here as $\mathrm{N}_{2}$ equivalent pressures.

An aging process was first carried out to obtain better emission stability and uniformity in the device. The FED to be analyzed was then connected to the main vacuum chamber through an opening set. Before the glass wall between the FE device and the vacuum system was broken, the FE properties of the device were measured in terms of the I-V characteristics and the current stability working at a constant anode voltage. The vacuum chamber and the opening sets were then pumped using the mechanical pump and the turbo molecular pump, and meanwhile, the vacuum chamber and the opening set were baked at approximately $250^{\circ} \mathrm{C}$ for 24 hours, to obtain a good vacuum level. When the baking temperature was reduced to room temperature, valve $\mathrm{V}_{2}$ was shut off,

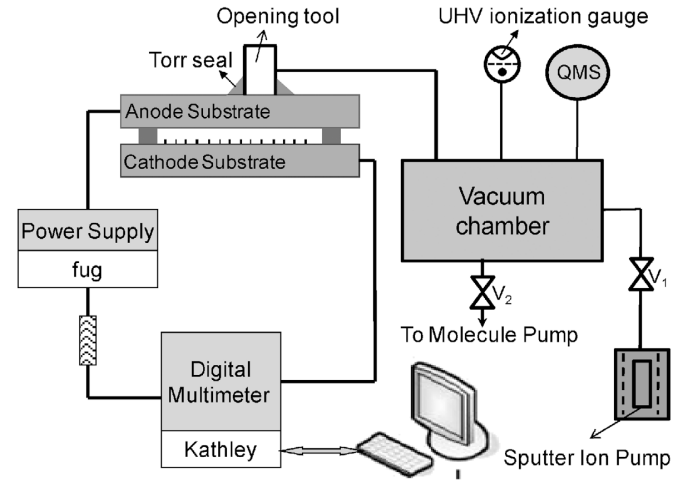

Fig. 2 Schematic diagram of the experimental system.

and the system was pumped down using the ion sputter pump. The final gas pressure in the system was $2 \times 10^{-6}$ $\mathrm{Pa}$. The residual gas spectra were recorded using the QMS mounted on the vacuum chamber. The device was then broken by the opening set, and the residual gases in the device diffused into the vacuum system. The residual gases were measured from the QMS mass spectra obtained before and after the breaking of the device. After being broken, the FE device became a part of the vacuum system that could be pumped on using the ion sputter pump. Thus, the outgassing components of the device under operation could be recognized using the QMS.

After an ultra high vacuum was obtained in the whole system, the FE properties and the emission current stability were measured and compared with those measured in the real device, where the gas pressure was much higher.

Figure 3 shows the emission current stabilities of the $\mathrm{CNT}$ and $\mathrm{ZnO}$ cathodes, as measured in the sealed FE device, and after the device was opened to the UHV chamber. After continuous operation at a constant anode voltage of $1150 \mathrm{~V}$ in the devices for 4 hours, the emission currents reduced from $47 \mu \mathrm{A}$ to approximately $19.7 \mu \mathrm{A}$ for CNT cathode, and from $50 \mu \mathrm{A}$ to $15.3 \mu \mathrm{A}$ for the $\mathrm{ZnO}$ cathode also at an anode voltage of $1150 \mathrm{~V}$. After the device was opened to the UHV system, the anode voltage was applied at $1100 \mathrm{~V}$ for CNT cathode and $1030 \mathrm{~V}$ for $\mathrm{ZnO}$ cathode, so that the initial emission current was close to the value tested in the device. This means lower anode voltage was needed to obtain the same emission current. And experimental data showed 


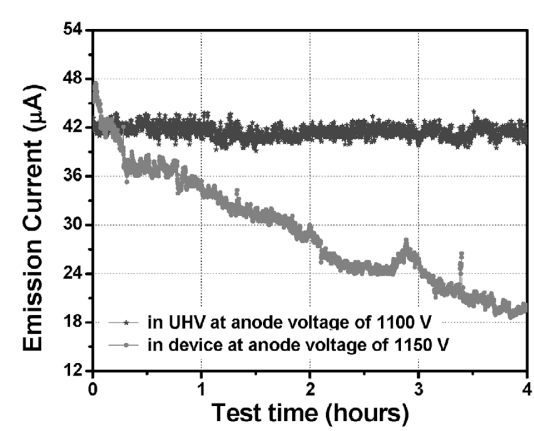

(a)

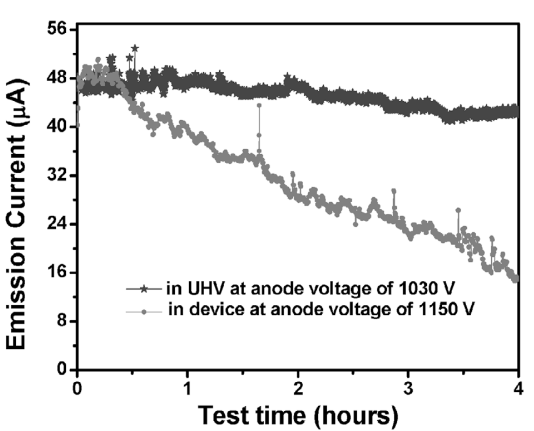

(b)

Fig. 3 Current stability of CNT (a), and ZnO (b) FEs in the device, and under UHV conditions.

that lower current degradation was achieved for the same cathode tested in the UHV system. The emission currents reduced from $42.5 \mu \mathrm{A}$ to approximately 41.4 $\mu \mathrm{A}$ for the CNT cathode, and from $48 \mu \mathrm{A}$ to approximately $42.5 \mu \mathrm{A}$ for the $\mathrm{ZnO}$ cathode, over a period of 4 hours. It was therefore clear that the emission current degradation was much more significant in the sealed FE device than under UHV conditions. The fact that all of the working conditions were kept constant except the gas environment meant that the improvement in the emission current stability was mainly due to the changes in the ambient gases of the cathode. We therefore concluded that the residual gases within the device did in fact impact the emission current stability and the lifetime of the cathodes. Because the gas pressure is higher in real sealed devices than in the UHV system, the presence of more residual gases lead to the degradation of the emission current. The residual gases in the device affect the emission properties in the several ways: First, the adsorption of residual gas molecules on the surface of the emitter increases the work function of the cathode ${ }^{15)}$, and influences the emission current of the cathode. Second, ambient gas molecules can be ionized through collisions with electrons as they (the electrons) are emitted and move to the anode. The ionized positive charges bombard the cathode under the low applied electric field, and cause ion sputtering and injection at the surface of the cathode, which also changes the work function and geometrical structure at the cathode surface ${ }^{16)}$. Finally, chemical reactions occur between the cathode material and the residual gases ${ }^{17}$, which results in permanent emission current degradation of the cathode. Therefore, more stable currents can be obtained by lowering the residual gas pressure in the device, and more reliable devices can be achieved by enhancing the stability of the cathode material and avoiding the interactions between the cathode materials and ambient gases that can affect the field emission character of the cathodes.

To investigate the residual gases in sealed $\mathrm{ZnO}-\mathrm{FE}$ and CNT-FE devices, the gases must be introduced into the vacuum chamber, where they can be distinguished by the QMS. Typically, this task is accomplished by making a hole in the glass plate, and permitting the gases to enter the chamber. The residual gas components can then be determined by comparing the gas spectra in the vacuum
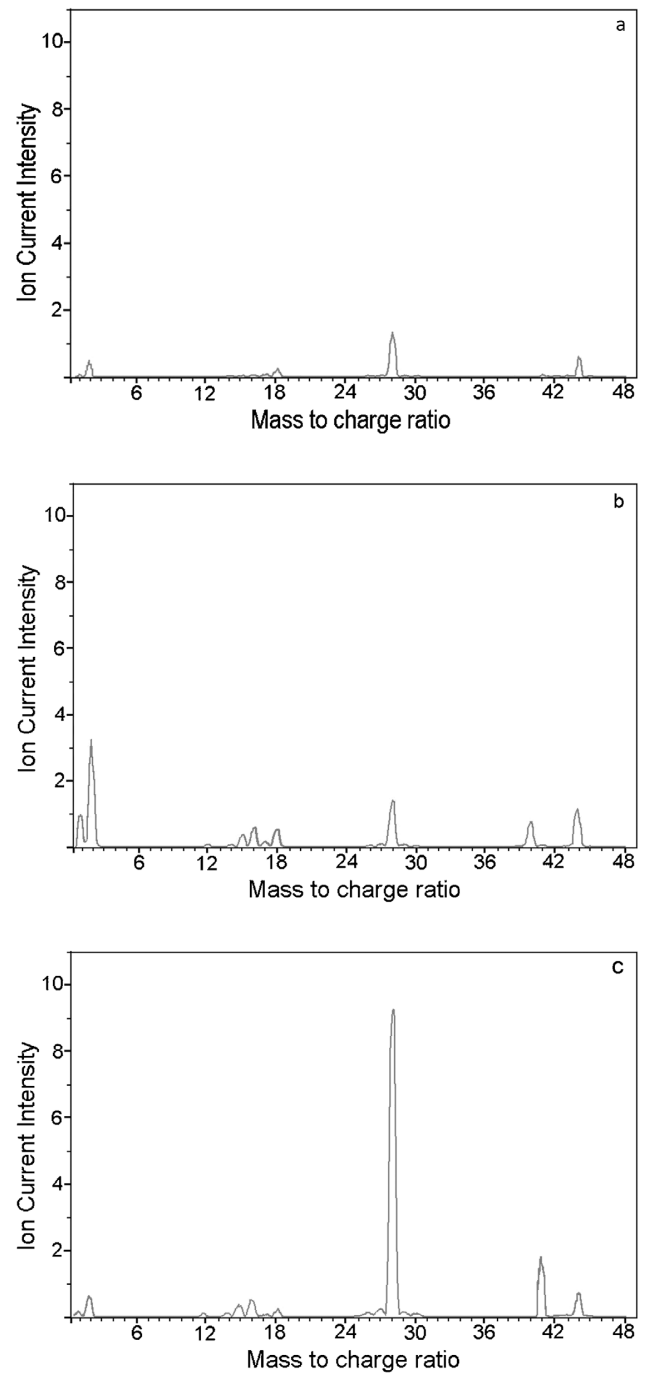

Fig. 4 Mass spectra in the vacuum chamber before the CNT-FE device was opened (a), after the device was opened (b), and when the device was working (c).

chamber before and after the device was opened to the system.

Figure 4 shows the mass spectra in the vacuum chamber before the CNT-FE device was opened, after the device was opened, and when the device was working after being opened. From the mass spectra in the vacuum 
chamber at these different stages, it was concluded that the residual gases in the sealed CNT-FE device were $\mathrm{H}_{2}$, $\mathrm{CH}_{4}, \mathrm{CO}, \mathrm{Ar}$, and $\mathrm{CO}_{2}$; some $\mathrm{C}$ was also observed in the outgassing components of the CNT-FE device. As shown in the mass spectra in Fig. 4(c), an increase in the $\mathrm{C}$ contents was observed during the operation of the CNT-FE device. This indicated the decomposition of the CNTs or organic components during electron emission, or under ion bombardment.

For the sealed $\mathrm{ZnO}-\mathrm{FE}$ device, the residual gases were $\mathrm{H}_{2}, \mathrm{CH}_{4}, \mathrm{CO}, \mathrm{Ar}$, and $\mathrm{CO}_{2}$, and the outgassing components were composed of $\mathrm{H}_{2}, \mathrm{CH}_{4}, \mathrm{CO}, \mathrm{O}_{2}, \mathrm{Ar}$, and $\mathrm{CO}_{2}$, as shown in Fig. 5. From the mass spectra shown in Fig. $5(\mathrm{c})$, large amounts of $\mathrm{O}_{2}$ were observed during the operation of the $\mathrm{ZnO}-\mathrm{FE}$ device. This component can be produced by the decomposition of $\mathrm{ZnO}$-or the decomposition of organic components in the $\mathrm{ZnO}$ paste-under ion bombardment ${ }^{18)}$. Outgassing $\mathrm{O}_{2}$ components were not observed among the residual gases for the $\mathrm{ZnO}-\mathrm{FE}$ device. The outgassing mass spectra were measured using QMS. The active component of $\mathrm{O}_{2}$ was finally pumped away using the getter in the device, and thus $\mathrm{O}_{2}$ was not observed in the residual gases.
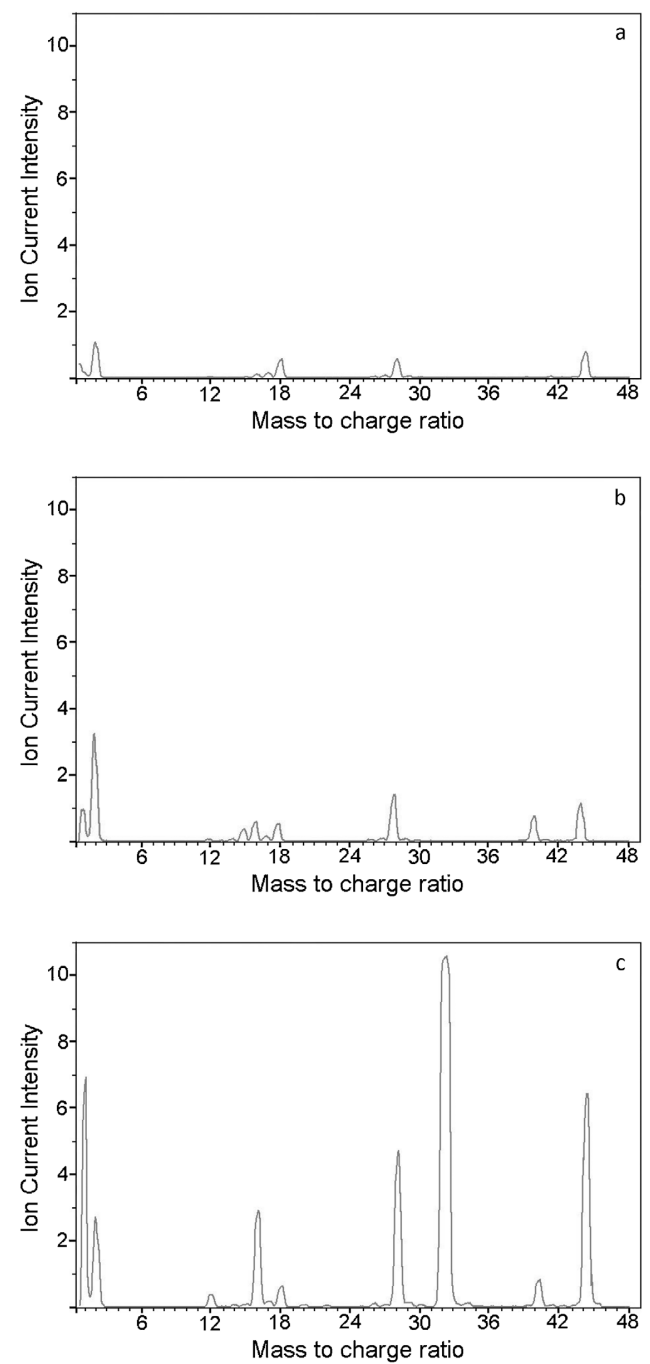

Fig. 5 Mass spectra in the vacuum chamber before the $\mathrm{ZnO}-\mathrm{FE}$ device was opened (a), after the device was opened (b), and when the device was working (c).
According to the analysis above, it is clear that the residual gases and outgassing components were different for the FE devices using MWCNT emitters and those using tetrapod $\mathrm{ZnO}$ emitters. These differences were mainly due to the interaction between the different cathode materials and the ambient gases during the fabrication or operation processes of the device. In the CNT-FE device, where carbon was abundant-due to the disassociation of the CNTs or non-graphitic carbon in the CNT paste, or the evaporation of the CNTs during electron emission ${ }^{19)}$-chemical reactions between $\mathrm{C}$ and $\mathrm{CO}_{2}$ or $\mathrm{O}_{2}$ would have led to increases in the amount of $\mathrm{CO}$ in the device using a CNT cathode. This would have resulted in an absence of $\mathrm{CO}_{2}$ in the CNT-FE devices. During the fabrication or operation of the $\mathrm{ZnO}-\mathrm{FE}$ device, unstable $\mathrm{ZnO}$ would have decomposed under heat or ion bombardment. The oxygen ions would then have recombined with carbon monoxide to create new $\mathrm{CO}_{2}$ molecules, as shown in equation (1). Chemical reactions between $\mathrm{CO}$ and $\mathrm{ZnO}$ would also have resulted in decreases in the amount of $\mathrm{CO}$, and increases in the amount of $\mathrm{CO}_{2}$, as shown in equation (2). These kinds of interactions between the ambient gases and cathode materials were directly responsible for the different residual gases measured in the FE devices when different cathode materials were used.

$$
\begin{aligned}
& \mathrm{ZnO} \stackrel{\text { decompose }}{\longrightarrow} \mathrm{Zn}^{2+}+\mathrm{O}^{2-} \\
& \mathrm{CO}+\mathrm{O}^{2-} \longrightarrow \mathrm{CO}_{2}+2 e \\
& \mathrm{ZnO}+\mathrm{CO} \longrightarrow \mathrm{Zn}+\mathrm{CO}_{2}
\end{aligned}
$$

Figure 6 shows the field emission properties of the CNTs and $\mathrm{ZnO}$ before and after the device was opened to the UHV system. For the CNT cathode, the turn-on voltage measured in the sealed device was $700 \mathrm{~V}$ (at a current of $0.5 \mu \mathrm{A}$ ), and this decreased to $530 \mathrm{~V}$ under UHV conditions. The same results were found for the $\mathrm{ZnO}$ cathode; the turn-on voltage reduced from $800 \mathrm{~V}$ in the sealed device to $700 \mathrm{~V}$ under UHV conditions. This result showed that the residual gases in sealed FE devices influence the field emission properties of the CNT and $\mathrm{ZnO}$ cathodes in another aspect.

From equation (1), it is clear that $\ln \left(I / V^{2}\right)$ is linearly related to $1 / V$. The slope and intercept of the $\mathrm{FN}$ proximity curve based on the data plots is decided by the work function $(\Phi)$ at the surface of the cathode and field enhancement factor $(\beta)$. Their relationships can be described as following functions,

$$
\left\{\begin{array}{l}
\text { intercept }=\ln \left(A a \frac{\beta^{2}}{\Phi}\right) \\
\text { slop }=-B\left(\frac{\Phi^{3 / 2}}{\beta}\right)
\end{array}\right.
$$

The intercept and slop of the FN plot in Fig. 6(b) and (d) are shown in table 1. Put these data into equation (4), the change of work function and geometrical factor can be obtained which was also shown in Table 1. This result shows that geometrical factor reduced for both kinds of cathode after being exposed to UHV condition. The 

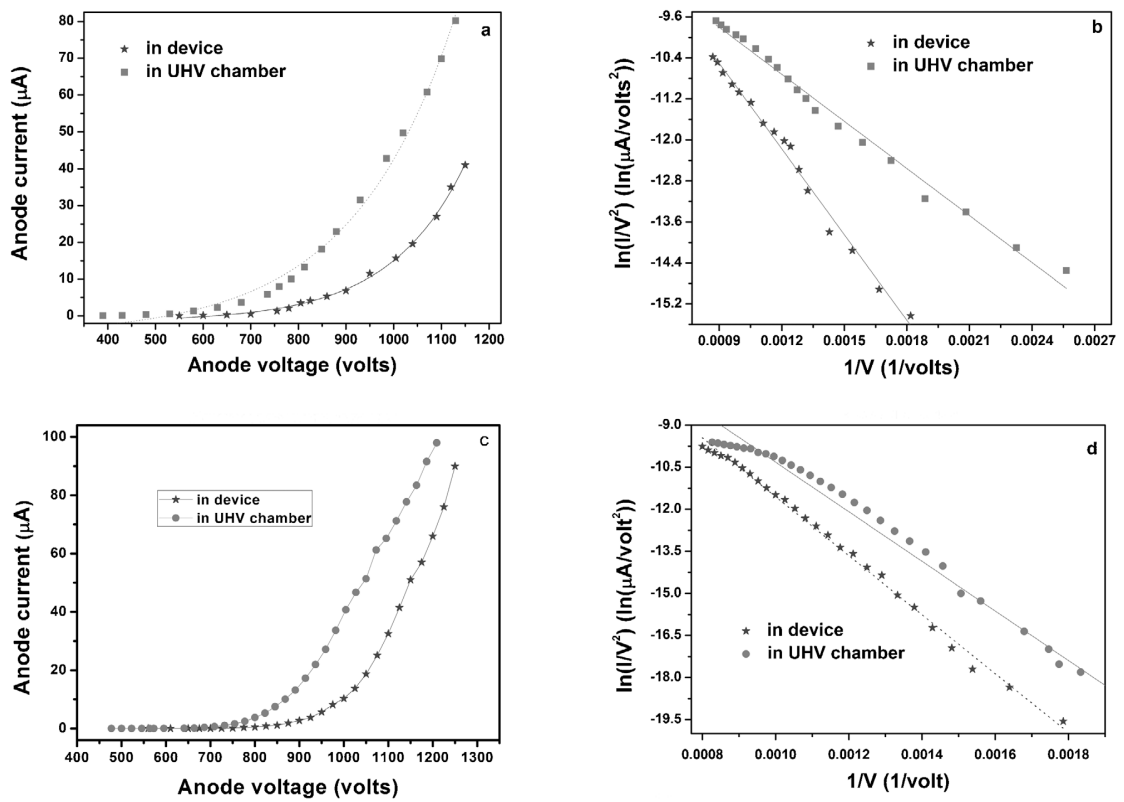

Fig. 6 IV (a), and FN (b) curves for the CNT cathode, and IV (c), and FN (d) curves for the ZnO cathode, in the sealed device and in the UHV chamber.

Table 1 Relationship between FN characteristics and work function or geometrical factor.

\begin{tabular}{l|c|c|c|c}
\hline \hline \multirow{2}{*}{} & \multicolumn{2}{|c|}{ CNT } & \multicolumn{2}{c}{ ZnO } \\
\cline { 2 - 5 } & Device & UHV & Device & UHV \\
\hline Intercept & -5.5 & -7.0 & -1.04 & -1.75 \\
\hline Slope & -5570 & -3060 & -10509 & -8583 \\
\hline Work function & \multicolumn{2}{|l}{$\Phi_{C N T-\text { device }} \approx 3.8 \Phi_{C N T-U H V}$} & $\Phi_{\text {ZnO-device }} \approx 2.4 \Phi_{\text {ZnO-UHV }}$ \\
\hline Geometrical factor & \multicolumn{3}{|c}{$\beta_{C N T-\text { device }} \approx 7.5 \beta_{C N T-U H V}$} & \multicolumn{2}{c}{$\beta_{\text {ZnO-device }} \approx 1.8 \beta_{\text {ZnO-UHV }}$} \\
\hline
\end{tabular}

reduction of geometrical factor will lead to lower emission current. But opposite experimental result has been displayed in Fig. 3. This is mainly attributed to the reduction of work function, which will make it easier to emit electrons.

According to the above discussions, the residual gases in sealed FE devices had obvious effects on the reliability and lifetime of both kinds of cathode materials. Residual gases absorbed on the surface of the cathode and raised its work function. Even the increase of geometrical factor can not balance this degradation. The ambient gas conditions were achieved by making a hole in the device to let gas into the UHV chamber. That is not to say that each kind of residual gas degraded the emission current for the CNTs and ZnO; the degradation of the emission current in the sealed FE devices resulted from the combined effects of all of the ambient gases around the cathode.

\section{Summary and Conclusions}

The emission current degradation of the same cathode was measured under different vacuum conditions, and it was proposed that the residual gases were responsible for the emission current degradation of the CNT and $\mathrm{ZnO}$ cathodes in sealed FE devices. The residual gases inside the sealed CNT FE device were $\mathrm{H}_{2}, \mathrm{CH}_{4}, \mathrm{CO}$, and $\mathrm{Ar}$, while in the $\mathrm{ZnO} F E$ device they were $\mathrm{H}_{2}, \mathrm{CH}_{4}, \mathrm{CO}, \mathrm{Ar}$, and $\mathrm{CO}_{2}$. The different cathode materials were directly responsible for the different gas components in the devices. These residual gases changed the work function at the surface of the cathodes through adsorption or ion bombardment, and thus degraded the emission current of the cathodes. Therefore, more reliable devices could be achieved by enhancing the stability of cathode material, and avoiding the interactions between the cathode materials and ambient gases that can affect the field emission character of the cathodes.

\section{Acknowledgments}

This work is supported by Key Project of He'nan Educational Committeeand (Grant no. 13A510263), and Luoyang Technology Development Project (Grant no. 1301009A).

\section{References}

1) Y. Q. Chang, X. H. Chen, H. Z. Zhang, W. J. Qiang and Y. Long: J. Vac. Sci. Technol. B, 25 (2007) 1249.

2) O. Yilmazoglu, R. Joshi, A. Popp, D. Pavlidis and J. J. Schneider: J. Vac. Sci. Technol. B, 29 (2011) $02 B 106$.

3) L. F. Chen, Y. H. Mi, H. L. Ni, Z. G. Ji, J. H. Xi, X. D. Pi and H. F. Zhao: J. Vac. Sci. Technol. B, 29 (2011) 041003.

4) R. H. Fowler and L. W. Nordheim: Proc. Roy. Soc., A119 (1928) 173.

5) S. K. Kang, J. H. Choi, J. H. Park, J. H. Han, J. B. Yoo, J. W. Nam, C. K. Lee and J. M. Kim: J. Vac. Sci. Technol. B, 22 (2004) 1345.

6) J. M. Bonard, J. P. Salvetat, T. Stockli, W. A. Heer, L. Forro and A. Chatelain: Appl. Phys. Lett., 73 (1998) 918.

7) J. M. Bonard, C. Klinke, K. A. Dean and B. F. Coll: Phys. Rev. B, 67 (2003) 115406.

8) L. Nilsson, O. Groening, P. Groening and L. Schlapbach: Appl. Phys. Lett., 79 (2001) 1036.

9) J. Xiao, G. M. Zhang, X. Bai, L. G. Yu, X. Y. Zhao and D. Z. Guo: Vacuum, 83 (2009) 265. 
10) Y. Wei, B. R. Chalamala, B. G. Smith and C. W. Penn: J. Vac. Sci. Technol. B, 17 (1999) 233.

11) Y. C. Ko and D. Jeon: Appl. Surf. Sci., 215 (2003) 228.

12) M. J. Gilkes, D. Nicolaescu and P. R. Wilshaw: J. Vac. Sci. Technol. B, 18 (2000) 948.

13) B. R. Chalamala, R. M. Wallace and B. E. Gnade: J. Vac. Sci. Technol. B, 16 (1998) 3073.

14) K. A. Dean, T. P. Burgin and B. R. Chalamala: Appl. Phys. Lett., 79 (2001) 1873.

15) Z. Li and C. Y. Wang: Chem. Phys., 330 (2006) 417.
16) V. S. Bormashov, A. S. Baturin, K. N. Nikolskiy, R. G. Tchesov and E. P. Sheshin: Nucl. Instrum. Methods Phys. Res. A, 558 (2006) 256.

17) Y. H. Yang, C. X. Wang, B. Wang, N. S. Xu and G. W. Yang: Chem. Phys. Lett., 403 (2005) 248.

18) Y. K. Cui, X. B. Zhang, W. Lei, Y. S. Di, M. Xiao, X. X. Yang and Z. W. Zhao: IEEE Electron Device Letters, 31 (2010) 479.

19) D. H. Kim, H. S. Jang, S. Y. Lee and H. R. Lee: Nanotechnology, 15 (2004) 1433. 